advanced disease. An acute respiratory ward and an innovative One Stop Palliative Care COPD clinic was used to test the theory that early palliative care intervention supports self-management, advance care planning and reduces avoidable hospital admissions.

Methods The introduction of a weekly Gold Standard Framework (GSF) meeting and the use of GSF prognostic indicators were initiated on a respiratory ward, to identify COPD patients in the last year of life. Patients were offered palliative care support, in hospital and community, to help them live well with the disease. A One Stop Palliative Care COPD clinic was introduced to promote self-management and advance care planning.

Results

- Hospital referrals have increased from 20\% (Aug18-Jan 19) to $62 \%$ (Feb 19-July 19)

- Community palliative care support has increased from $21 \%$ to $70 \%$

- Increase in documented advance care plans has increased from $46 \%$ to $90 \%$

- $77 \%$ of patients were identified and referred for inclusion on the GSF register

- Patients were empowered to live well with the disease

- Outcome measures have shown that COPD patients have a greater symptom burden than other patients referred to palliative care.

- The project can be replicated across all disease groups

Conclusion This model has validated the theory that early referral to palliative care services for COPD patients in the last year of life, enhances patient care. It encourages and promotes living well with the disease, advance care planning with the intention of reducing avoidable hospital admissions.

\section{RESPECT, FIND OUT WHAT IT MEANS TO ME- AN AUDIT OF EMERGENCY TREATMENT PLANS}

Matthew Murden, Roop Gill, Sinead Henderson. Ashford and St Peter's NHS Trust

\subsection{6/spcare-2020-PCC.161}

Introduction ReSPECT is a national initiative led by the Resuscitation Council(UK). It encourages doctors to work in partnership with patients to establish their wishes and thoughts on future treatments should they become unwell. Moving away from old DNAR forms requires a significant culture shift. We replaced these forms at Ashford \& St Peter's Hospital NHS Trust November 2018. In this audit we aimed to assess the success of the rollout of ReSPECT forms hospital-wide in the first 3 months, aiming for more than $98 \%$ of patients having a valid form.

Methods Retrospective notes audit. The first 10 patients discharged from each ward, including patients who died, were selected for an electronic notes review looking for a ReSPECT form or documented evidence that a ReSPECT form was filled during admission. The presence of a ReSPECT form and the extent to which it was correctly filled out were recorded in a spreadsheet.

Results 209 patient records were examined (100 Male, 109 Female) across a mix of surgical and medical wards, average age of 77 years. A total of 87 forms were reviewed, meaning
42\% had a ReSPECT form present. Personal/clinical details and summary of emergency care and treatment recommendations were completed $70 \%$ of the time. Areas for improvement include mental capacity documentation and section 3 patient personal preferences.

Conclusions Although uptake initially appears poor in retrospect our standard of $98 \%$ was likely too high, a $42 \%$ uptake is a significant achievement for the first 3 months. By identifying areas for improvement we have been able to put in place initiatives to target these specifically. We recognise the need to continue to facilitate a change in culture within our trust and promote shared decision making and 'the conversation' as a process not a one off. We will reaudit next year.

\section{ANALYSIS AND IMPACT OF NON MEDICAL PRESCRIBING INTERVENTIONS AT JOHN TAYLOR HOSPICE}

T Nayyar, S Begum, C Hirsch. John Taylor Hospice, University of Birmingham

\subsection{6/spcare-2020-PCC. 162}

Introduction The growth in non-medical prescribing (NMP) by nurses and pharmacists has been well documented across a range of medical specialities. Prescribing by allied health professionals in palliative care is a relatively recent phenomenon. Outcome data for community palliative care prescribing is poorly characterised, and evidence of impact remains unsubstantiated. The purpose of this study was to gauge self-reported outcomes of prescribing interventions by nurse and pharmacist prescribers for patients in a community setting.

Methods Prescribing data was collected between 1st May and 1st July 2019. Data included perceived reasons for prescription issue and the outcome of the intervention. Data was anonymised and analysed using MS Excel to quantify the classes of drugs prescribed, and identify prescribing patterns. A second phase involved an online survey of medical and NMPs at the hospice to explore their views on independent prescribing.

Results Data from 7 nurse and 2 pharmacist NMPs were analysed. In over one third (35\%) of prescribing events, prescriptions were for routine symptom control such as pain or nausea. Provision of anticipatory injectable medicines was the second most common reason for prescription issue. Forty eight percent of patients prescribed medicines for symptom control achieved faster access to treatment. In $57 \%$ of prescription events a GP home visit was prevented. Analysis of the surveys is pending and themes arising from these will be shared amongst the hospice prescribing team.

Conclusion As a result of non-medical prescribing, patients receiving community palliative care were able to access crucial medicines in a timely manner which may have facilitated better symptom control. The study's main limitation was that prescribers completed data collection forms themselves, which may have influenced their choice of outcome. Potential savings of approximately $£ 2,595$ in GP visits suggest that utilisation of NMP prescribing should be more widely considered in palliative care teams. 